HERAUSGEBER:

Geh. San.-Rat Prof. Dr. Schwalbe

Berlin-Charlottenburg, Schlüterstr. 53
VERLAG:

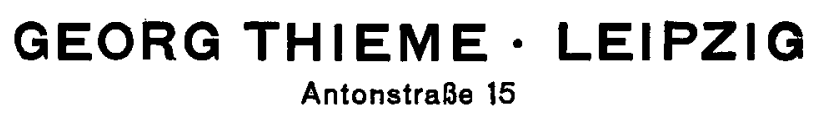

\section{Erwiderung auf die Abwehr des Herrn Geheimrat Kirchner in Sachen des Friedmannschen Tuberkuloseheilmittels. ${ }^{1}$ )}

Von Dr. Friedrich Franz Friedmann,

ao. Professor an der Universität Berlin.

Herr Geh.-Rat $\mathrm{Ki}$ r ch ner befindet sich im Irrtum, wenn er schreibt, daß ich ihn zuerst in der Tagespresse angegriffen hätte. Dem von ihm zitierten Artikel in Nr. 41 der ,Neuen Berliner 12 .Uhr" vom 27. Februar 1919 stand ich vollstandig fern und habe, als mir das Blatt am Morgen des 28. Februar vorgelegt wurde, sofort eine diesbezügliche Erklärung an die Redaktion der Zeitung geschickt, welche diese auch am Sonnabend den 1. März in Nr. 43 wie folgt abdruckte:

„Eine Zuschrift des Erflnders des Tuberkuloseheilmitteis. Zu unserem. Artikel schreibt uns Dr. Friedrich Franz Friedmann, der Erfinder des Tuberkulosehelimittels: „Soeben wird mir ein Exemplar der Nr.41 Ihrer Zeitung abergeben. Es liegt mir, der ich voliståndig aberrascht bin, daran, festzustellen, daB ich der in dem Artikei "Frfedmann, Wilson usw "gegebenen Veröffentlichung der angetärten Tatsachen "In dieser Form voliständig fernstehe. Ich darf Sie blten, dies freundlichst Ihren Lesern in der năchsten Nummer mittellen zu bitten, dies freundlichst Ihren Lesern in der năchsten Nummer mittellen zu
wolien." Wir steilen gerne fest, daß der Autor jenes Artikels Herrn Dr. Friedm a n n nicht kennt und Herr Dr. Fried ma $n$ n nach der ganzen Sachlage wede direkt noch indlrekt etwas davon wußte, daß ein Artikel tiber inn und gegen gewisse MiBstånde der Medizinalverwaltung in dieser oder jener Form erscheinen werde."

Demnach habe nicht ich Herrn $\mathrm{K}$ i r ch $\mathrm{ner}$, sondern dieser mich in der Tagespresse, und zwar in Nr. 90 der Nationalzeitung 8 Uhr-Abendblatt vom 22. April 1919, in einem Artikel „Das Tuberkuloseserum Dr. Friedmanns" angegriffen, in dem es wörtlich heißt:

„Friedman n führte sich sozusagen unganstig ein, Indem auf selne Veraniassung, ailerdings in Uebereinstimmung mit dem Leiter der dortigen Anstait, 1200 gesunde' $K$ Inder des Walsenhauses in Rummelsburg mit dem aus Kailtbiätern (Schlidkröten) gewonnenen Serum geimpft wurden. Es solite hierdurch die Ungiftigkeit' des Mitteis bewiesen werden."

Hierdurch veranlaßte. Herr $K$ i r c h n e r mich, ebenfalls in der Tagespresse diese Behauptung zu widerlegen. Ich erwiderte ihm in Nr. 91 der ,Nationalzeitung 8 Uhr-Abendblatt" vom 23. April 1919:

„Was die durch mich vorgenommene Prüfung auf Unschädlichkelt anbetrifft, so 1 st die Angabe Kirchners, ich hätte Kindergeimptt, um die Ungiftigkeit des Mitteis zu bewelsen, falsch. Die Un schädilchkeit habe ich viele Jahre vorher an Hunderten von Tieren festgestell und bevor Ich an die Hellimpfung beim Menschen ging, mir das Mittel selbst eingespritzt (1909). Erst nachdem ich mich an mehreren Hundert tuberkulös erkrankten Menschen von der hellenden Wirkung des Mittels liberzeugt hat te, hieit ich es 1911 fär an der Zelt, Schutzimpfungen vorzunehmen, und machte dem Leiter des Hospitais des Rummeisburger Waisenhauses, nachdem auch er Heilungen seiner tuberkulösen Patienten konstatlert hatte, diesen Vorschlag. Ich habe auch seiner tuberkuiosen Patienten konstatiert hat te, diesen Vorschlag. Ich habe auch nicht, wie Kirch ne r angibt, 1200, sondern 320 Kinder - die zum großen Teil Rummeisburger Hospital, die ibrigen in einem hiesigen Wöchnerinnenheim."

Ich wies also nach, daß der für einen Arzt ungeheuer schwere Vorwurf Ki r ch n r s, ich häte Kinder geimpft, um die Ungiftigkeit des Mittels zu beweisen, der Wahrheit widerspricht. Wenn der von $\mathrm{K}$ i r c h n e r zitierte ,Vorwärts" damals (15. November.1912) sicher aus ungenügender Information sich in dieser Hinsicht abfällig âußerte, so muß man sich fragen, wie es möglich ist, daß $\mathrm{K}$ i r c h n e r, der doch hierüber gut informiert ist, noch jetzt (22. April 1919) behauptet, daß ich die Ungiftigkeit des Mittels an Kindern hätte erproben wollen. Heute schreibt der ,Vorwärts“ (25. Juli 1919) von der ,Fortsetzung des schon lange von einer unter dem alten Regime allmächtigen wissenschaftlichen Clique geführten Unterdrückungskampfes, unter dem unter anderen ja auch Prof. F r i e d m a n n zu leiden hatte, der Finder des jetzt zu so hohen Ehren gekommenen Tuberkulosemittels, das solange von Ministerialdirektor $\mathrm{K}$ i r c h n e r bekämpft wurde."

Bedauerlich ist es auch, daß $\mathrm{K}$ i r ch $\mathrm{n}$ e r erst heute, der

2) In seinem Begleitschreiben bemerkt Herr ao. Prof. Friedmann: ,Ihrem Gerechtigkeitsgefühl überlasse ich es, ob Sie, da Sie Herrn Geheimrat Kirchners Aufsatz in meiner Sache an der Spitze der betreffenden Nummer publiziert haben, meiner Erwiderung dieselbe Stelle einrüumen." Die Erwiderung erscheint deshalb an der Spitze dieser Nummer.
Not gehorchend, die amtliche Feststellung vom 13. Februar 1913 bekanntgibt, nämlich:

,daß die im November 1911 in Rummelsburg geimpiten Kinder nach der Injektion monatelang in der Anstalt unter Beobachtung des Oberarztes geblieben wären, ohne daß sich eine krankhafte Erscheinung gezeigt hätte, und daß sie inzwischen bis auf zwei entlassen wären, daß von den 16 nach Berlin entlassenen Kindern am 9. Januar 1913 auf Ladung 10 erschienen wären, die von Prof. $M$ ülle $r$ untersucht und frei von Tuberkulose befunden wurden."

Auch jetzt sagt $\mathrm{K}$ i r c h n e r in seinem Artikel nur, daß in dem Berichte des Oberprăsidenten in Potsdam an den Minister des Innern vom 22. Dezember 1912, betr. meine Heilimpfungen in Rummelsburg ,,bei Kindern mit schwerer Knochentuberkulose eine wesentliche "Besserung der tuberkulösen Erkrankungen" vermerkt sei. Dagegen lautet die betreffende Stelle dieses Be- $^{-}$ richtes wörtlich:

„Nachdem bei diesen Kindern keinerlei Störung des Allgemeinbefindens, vielmehr eine wesentliche Besserung, zum Teil auch eine Heilung der schweren tuberkulösen Erkrankungen eingetreten war, hatte Dr. $M$ ù l l e r kein Bedenken, dem Dr. Friedmann Ende Oktober 1911 etwa 50 gesunde Kinder zur Schutzimpfung zu übergeben."

Ferner sagt $\mathrm{K}$ i r c h n e r jetzt, ich hãtte nur zu ihm $\mathrm{zu}$ kommen brauchen und eine Mitteilung des E h r li ch schen Berichts wäre erfolgt. Demgegenüber sei aus den Akten des Ministeriums (E h r l i c h scher Unschãdlichkeitsbericht vom 3. August 1913) folgender zugleich mit dem Bericht an den $\mathrm{Mi}$ nister gerichtete Antrag $\mathrm{Ehrlichs}$ wörtlich wiedergegeben:

„,Frankfurt a. M., 3. August 1913. Euerer Exzellenz gestatte ich mir in der Angelegenheit der Nachprüfung des F. F. F r i e d m a n n schen Tuberkulosemittels im AnschluB an den diesseitigen Bericht vom 31. Mai d. J. nach dem jetzigen Stande der Unschädlichkeitsprüfungen der Kultur im Tierversuch als vorläufiges Ergebnis beiliegenden Bericht sehr ergebenst zu überreichen. Herr Dr. F r i e d m a n n hat gelegentlich eines Besuches vor einigen Tagen die wohlberechtigte Bitte ausgesprochen, ihm die bisher hier erhobenen Be'unde zugänglich zu machen, und bitte ich Euer Exzellenz um Genehmigung dazu.

K i r c h n e r wußte also, daß nicht nur ich um den Bericht gebeten, sondern daß $\mathrm{Ehrlich}$ diesen meinen Wunsch als berechtigt anerkannt und befürwortet hatte; trotzdem haben weder ich noch die Aerztewelt den vorläufigen noch den endgültigen Unschädlichkeitsbericht erhalten. Ich habe die Ehrli ch schen Berichte nicht eher zu sehen bekommen, als bis sie mir der Nachfolger $\mathrm{K}$ irchners, Herr Geh.-Rat Got t s t e i n, jetzt vor wenigen Wochen zuganglich gemacht hat. $\left.{ }^{1}\right)$ Daß nicht nur die deutschen, sondern auch die ausländischen Aerzte damals auf die Bekanntgabe des Gutachtens warteten geht z. B. aus einem Schreiben des Staatsrats Dr. v. H e u k i n g,

1) La Kirchner mit den Worten, ich hätte das Gutachten ,auf irgendeine Weise erhalten ", den. Eindruck hervorrufen könnte, als ob ich mir die Kenntnis desselben auf unrechtmäBige Weise verschalft hätte, so sei bemerkt, daß meine Kenntnis des wesentlichen Inhalts der Ehrlichschen Unschädlichkeitsberichte aus folgenden Quellen stammt: 1. aus persönlichen mir gemachten Mitteilungen Ehrlichs; 2. aus dem Briefe eines Berliner Arztes vom 20. Juli 1914, der eine längere Unter. redung mit Herrn Geheimrat $A b e l$ im Ministerium des Innern wieder. gibt, wonach Geheimrat $A b e l$ an der Hand des Gutachtens, aus drücklich erklärte, daß Ehrlich in einer Versuchsreihe von über 100 Meerschweinchen durch die Friedmannschen Bazillen nicht ein einziges Mal eine Tuberkulose bei diesen so überaus empfindjichen Tieren erzeugen konnte"; 3. aus einem Briefe von Geheimrat Kraus an mich, vom 21. II. 1918 des Inhalts ,Ehrlich habe ihm 1914 ausdrücklich geschrieben, daB bei den in seinem Institut unter seiner Leitung an über 100 Versuchstieren mit der Friedmann.Kultur angestellten Versuchen irgendwelche tuberkuloseverdächtige Prozesse nie beobachtet wurden"; 4. aus einem Briefe des Hauptgeschäftsführers der Landwirtschaftskammer vom 8. VII. 1918, welcher die Ehrlichschen Gutachten im Ministerium gelesen hatte, in denen die ,völlige Unschädlichkeit des Friedmannschen Mittels beim Tierversuch konstatiert" sei. 
des Chefarztes eines großen Petersburger Hospitals*), hervor, in welchem es heißt:

,St. Petersburg, 1. April 1914.

Alle Einwände wären widerlegt, sobald das $E$ h rll c h sche Outachten in seinem Wortlaut veröffentlicht wlirde.

Auch daß Ki r c h n e r einer Abgesandten der New-Yorker Staatsregierung, Mrs. L o e b aus New York, die eigens nach Berlin gekommen war und $\mathrm{K}$ i r ch $\mathrm{n}$ e r aufsuchte, um sich über den stand der Fri e d m a n n schen Tuberkuloseangelegenheit in Berlin $\mathrm{zu}$ informieren und die $\mathrm{zu}$ ihm sagte:

Ich weiß, daß $\mathrm{E} h \mathrm{rli}$ ch die völlige Unschädlichkeit des Friedmannschen Mittels bestätigt hat; ich glaube, Sie haben die Pflicht, endlich das Outachten zu veröffentlichen."

die Auskunft verweigerte1) - ,es lag keine Veranlassung vor" — ist um so unbegreiflicher, als $\mathrm{ja} \mathrm{K}$ i r c h $\mathrm{ner}$, wenn er es noch nicht von selbst gewußt hätte, durch die von ihm jetzt angeführten Worte Ehrlichs über die ,internationale Bedeutung der Angelegenheit". belehrt worden war.

Nun zur Beantwortung der von $\mathrm{Ki} \mathrm{rehner}$ an Herrn Geh.-Rat $\mathrm{K} \mathrm{r} \mathrm{u} \mathrm{s} \mathrm{e}^{\mathbf{2}}$ ) und mich gestellten Frage:

,Was ich dazu sage, daß das Friedmannsche Mittel sowohl therapeutische als auch immunisierende Wirkungen bei mit Tuberkelbazillen infizierten Meerschweinchen vermissen lie $\beta q^{r c}$

Zunächst stelle ich fest, was auch aus dem von $\mathrm{K}$ i r c h $\mathrm{n}$ e r zitierten Briefe $\mathrm{Eh} \mathrm{rli} \mathrm{ch}$ s vom 12. Februar 1913 ohne weiteres hervorgeht: $\mathrm{K}$ i r c h n e r hatte mir geraten (oder mich beauftragt), E hrli ch die zur Herstellung des Mittels dienende Kultur zwecks Prüfung auf Schädlichkeit oder Unschădlichkeit beim Meerschweinchen zu übergeben. Niemals hat mir K i r c h $\mathrm{n}$ e $\mathrm{r}$ davon Kenntnis gegeben, daß diese Meerschweinchen, die Ehrlich ,durch Friedman n selbst zwecks Prüfung auf Unschädlichkeit hatte spritzen lassen" oder entsprechend behandelte Tiere daraufhin geprüft werden sollten, ob sie etwa einer Ueberschwemmung mit Hunderten von Millionen giftiger menschlicher Tuberkelbazillen standhielten. Ich hätte $\mathrm{K} \mathrm{i} \mathrm{r} \mathrm{c} \mathrm{h} \mathrm{-}$ $\mathrm{n}$ e $\mathrm{r}$ sonst selbstverständlich sofort nochmals mitgeteilt, daß dieses Beginnen in dieser Form zwecklos sei. In meinem von $\mathrm{K}$ i r c h n e r zitierten, ihm also bekannten Vortrage vor der Berliner medizinischen Gesellschaft ${ }^{8}$ ) heißt es wörtlich:

Zwar besitzen sie (die mit dem Mittel geimpften Meerschweinchen) der schweren künstlichen Infektion gegenubber keine volle Immunität, aber während tuberkulös gemachte unbehandelte Tiere nach durchschnittlich 110 Tagen erlagen, blieben dieselben durch nur eine einzige therapeutische Injektion dieses Mittels durchschnittlich 363 Tage, also fast ein Jahr lang am Leben. Und ebenso genügte eine immunisierende Injektion, um das Leben des vorbehandelten und später mit hochvirulenten menschlichen Bazillen infizierten Tieres über viermal so lang als dasjenige der Kontrollen, $d$. $h$. der nur mit menschlichen Bazillen infizierten Tiere, zu erhalten."

Die mit genügend großen Dosen des Friedmannschen Mittels behandelten Meerschweinchen zeigen tatsächlich bei Infektion mit sehr geringen Mengen virulenter Tuberkelbazillen, die aber genügen, um sämtliche nichtbehandelten Kontrollen an Tuberkulose zu töten einwandfrei immunisierenden und therapeutischen Effekt. H a n A r o n s o n, ein anerkannter Sachkundiger auf dem Gebiete der Tuberkuloseimmunisierung, bestätigt meine Angaben:4)

Jedoch hat Friedmann sicher bei einigen Meerschweinchen eine gute Widerstandsfähigkeit durch seine Vorbehandlung erzielt; zwei von ihm dem Laboratorium des KaiserFriedrich - Kinderkrankenhauses übergebene Meerschweinchen wurden zu gleicher Zeit mit einer größeren Zahl von Kontrolltieren subkutan mit Tuberkelbazillen infiziert. Die letzteren erlagen der Infektion sämtlich in drei bis fünf Monaten, während die geimpften Tiere noch nach einem Jahre vollig munter waren und sich in gutem Ernahrungszustande befanden.

Wenn es auch nur gelingt, einzelnen Meerschweinchen einen solchen Impfschutz zu verleihen, so ist dies immerhin als ein großer Erfolg zu bezeichnen. Denn jeder, der sich mit Immunisierungsversuchen am Meerschweinchen beschafftigt hat, weiß, wie außerordentlich selten und schwer eine solche Widerstandskraft zu erzielen ist."

Nach Selte rs Schätzung ${ }^{5}$ ) enthält, $1 \mathrm{mg}$ feuchte Tuberkelbazillenmasse mindestens 1 Milliarde Einzelbazillen“. Die von $\mathrm{K} \mathrm{i} \mathrm{r} \mathrm{c} \mathrm{h} \mathrm{n} \mathrm{e} \mathrm{r} \mathrm{angegebene} \mathrm{für} \mathrm{je} \mathrm{ein} \mathrm{Frankfurter} \mathrm{Meerschweinchen}$ gewählte ,,schwache Dosis des Typus humanus $0,5 \mathrm{ccm} 1 / 1000$ ", d. h. $1 / \mathrm{mg}$ Tuberkelbazillen-Reinkulturmasse, enthielt demnach 500000000 Bazillen. Da nun nach den mühevollen Unter-

*) Dr. v. Heuking war nicht Chefarzt, sondern Leitender Arzt der Chirurgischen Abteilung des Kinderhospitals des Prinzen von Oldenburg in Petersburg.

D. Red.

1) Auch das mir andauernd vorgehaltene amerikanische ,Verbot" wäre damals natürlich längst aufgehoben gewesen, wenn die Ehrlichschen offiziellen Unschädlichkeitsberichte in Amerika bekannt gewesen wären. - Herr Geheimrat $K$ ruse ermächtigt mich auf die diesbezügliche Frage Kirchners zu der Antwort, daß er mit umfangreichen Immunisierungsversuchen beschäftigt ist, über die zu gegebener Zeit berichtet werden wird. - ${ }^{8}$ ) B. kl. W. 1912 Nr. 47. - $)$ D. m. W. 1914 Nr. 10. - ) Vöff. der Robert Koch.Stiftung H. 11 u. 12. suchungen und Berechnungen von Geh.-Rat F rae $\mathrm{kel}^{1}$ ) in Halle ein einziger virulenter Tuberkelbazillus meistens zur Meerschweininfektion ausreicht, so sind die von $K \mathrm{irch}$ e $\mathrm{r}$ zitierten Frankfurter Meerschweinchen statt mit der bei den Kontrollen tödliche Tuberkulose erzeugenden Minimaldosis - wie es zur Feststellung eines immunisatorischen und therapeutischen Effektes selbstverständlich notwendig gewesen wäre - mit der etwa 500000000 mal so großen Menge, ,geprüft" worden. $\mathrm{Eh} \mathrm{rlich} \mathrm{selbst} \mathrm{bezeichnet} \mathrm{ja} \mathrm{auch} \mathrm{in} \mathrm{seinem} \mathrm{Bericht} \mathrm{an} \mathrm{den}$ Minister diese Versuche als ,nebensächlich".

Auch Prof. Selt e r (Königsberg) sagt:

„Für beide Fragen — ob es eine Immunităt bei Tuberkulose gibt und welcher Art sie ist - sind im wesentlichen Meerschweinchen als Versuchstiere benutzt worden, deren Empfänglichkeit jedoch so hoch ist, daß schon einige wenige Bazillen eine tödliche Infektion hervorrufen. Diese tödliche Minimaldosis müßte aber erst mal festgelegt werden, ehe man an die Lösung so schwieiger Fragen gehen darf.

Daß nicht nur bei Meerschweinchen mit menschlichen Tuberkelbazillen, sondern auch in der widerstandsfăhigen Kaninchenhornhaut mit dem Typus bovinus des Tuberkelbazillus durch Impfung von nur ganz wenigen (nach $\mathrm{P}$. H. R o m er berechnet etwa vier) Perlsuchtbazillen schon ein fortschreitender tuberkulöser Krankheitsprozeß erzeugt wird, geht aus den ganz exakten quantitativen Feststellungen von $\mathrm{K}$ r u s i $\mathrm{s}^{2}$ ) hervor.

Es gibt eben keine absolute Immunität: Jede Immunität. ob gegen Pocken oder Scharlach, gegen Diphtherie oder Tuberkulose, ist nur relativ und darum durchbrechbar.

Aber abgesehen davon, daß der therapeutische und immunisatorische Effekt des Friedmannschen Mittels im Meerschweinversuch bei Verwendung kleinster, aber für Kontrollen tödlicher Bazillenmengen erwiesen ist, wird der Wert solcher Feststellung für die menschliche Pathologie vielfach überschätzt, weil nämlich die Art der künstlichen Meerschweinchentuberkulose und die der spontanen menschlichen Tuberkulose in keiner Weise in Analogie gesetzt werden kann. v. B e h r i n g sagt:

Bekanntlich unterliegen Meerschweinchen und andere Tiere nach der Einführung virulenter Tuberkelbazillen unter die Haut in die Bauchhohle und in die Blutbahn einem KrankheitsprozeB, der gar keine Aehnlichkeit besitzt mit der menschlichen Lungenschwindsucht,"

Ebenso betont $\mathrm{M}$ a ragli a n o den

,großen Unterschied zwischen der natürlichen Infektion des Menschen und der experimentellen des Meerschweinchens, da wir im ersten Falle gegen keine so große Menge von auf einmal eingedrungenen virulenten Bazillen zu kâmpfen haben, wie es der Fall ist, wenn ein Meerschweinchen experimentell infiziert wird,"

und ferner,

„daß normalerweise bei dem Menschen die Infektion nicht so stürmisch stattfindet wie bei der experimentellen Tuberkulose der Tiere."

Wenn Ki r ch n e r sagt, meine Behauptung, daB die Worte ,nicht etwa aber, daß günstige Resultate damit erzielt worden sind" nachweislich nicht $\mathrm{zu}$ dem Ehrlich schen Gutachten gehörten, sondern von $\mathrm{K}$ i r c h n e r selbst hinzugefügt worden sind, widerspreche der Wahrheit, so erkläre ich hierzu, daß $\mathrm{Ki}$ r ch n e r im ,8 Uhr-Abendblatt" die wesentliche Tatsache, daß sich nämlich diese von ihm sogenannten ungünstigen Resultate lediglich auf die mit millionenfach zu hoher Infektionsdosis infizierten Meerschweinchen beziehen fortgelassen hat, und erkläre ferner, daß $\mathrm{Eh}$ r li c h diese sogenannten Immunisierungs- und therapeutischen Versuche mit den Ueberdosierungen, wie bereits erwähnt, für "nebensächlich" und nur die Unschädlichkeitsversuche für ,,abgeschlossen“ erklärt hat, K i r c h n e r dagegen im „8 Uhr-Abendblatt" den Anschein erweckt hat, als ob auch diese Immunisierungs- und therapeutischen Versuche von $\mathrm{Eh} \mathrm{rli} \mathrm{ch}$ als maßgebend hingestellt seien. Der Schlußbericht $\mathrm{Eh} \mathrm{r} \mathrm{li} \mathrm{chs} \mathrm{an} \mathrm{den} \mathrm{Minister}$ endet mit dem Satze:

Ich glaube hiermit die Nachprüfung des Friedmannschen Mittels betreffend Unschädlichkeit im Tierversuch als abgeschlossen erklären zu können."

Was das von $\mathrm{K}$ i r c h n e r erwahnte Tuberkulin anbetrifft, so habe auch ich stets betont, daB die $\mathrm{K}$ o $\mathrm{ch}$ sche Entdeckung des Tuberkulins als diejenige des ersten spezifisch-elektiven Bakterienproduktes einer der wichtigsten Schritte für die Entwicklung der Immunotherapie war. Darum darf aber das Rad der Entwicklung nicht da stillstehen, wo $K$ o $c$ h s überragende Forschungen aufgehört haben, und wenn $\mathrm{Kirch}$ e r und seine Freunde im Falle des Friedmannschen Mittels an ein Tuberkulosemittel die Anforderung stellen, daß es Meerschweinchen gegen künstliche Infektion mit Hunderten von Millionen virulenter Tuberkelbazillen immunisieren und derartig infizierte Tiere heilen müsse, so sei demgegenüber daran erinnert, daB das von $\mathrm{K}$ i r c h n e r erwähnte Tuberkulin wede

1) Fraenkel u. Baumann, Untersuchungen über die Infektiosi tät verschiedener Kulturen des Tuberkelbazillus, Zschr. f. Hyg. 54

I) Krusius, Experim. Tuberkulosestudien. Vöff. d. Robert KochS K r usius, E
Stiftung 1912 H. $5 / 7$. 
Meerschweinchen gegen die geringste Infektionsdosis immunisiert, noch derartig infizierte Meerschweinchen heilt.

Die Frage, ob das Friedmannsche Mittel mehr leistet als das Tuberkulin, möchte ich nicht erörtern: darüber haben årztliche Autoren, die beide Behandlungsmethoden jahrelang ernst geprüft haben, entschieden.

Wenn $\mathrm{K}$ i r c h n e r sodann einige unter den in Frankfurt geimpften Meerschweinchen hervorhebt, die an vermeintlicher Vergiftung eingegangen sind, so wei $B$ ein jeder, der viel mit Meerschweinchen arbeitet, daß diese Tiere nicht nur oft an bekannten Seuchen erkranken und sterben, sondern auch oft hinsiechen, ohne daß man die geringste Ursache dafür nachweisen kann. Auch Ehrli ch selbst klagt ja schon in seinem vorläufigen Bericht vom 4. August 1913, daß

,die Nachprüfungsergebnisse leider stark beeinträchtigt sind durch die in unseren Stallungen in den Frühjahrsmonaten wie gewöhnlich herrschenden Seuchen" 1914:

und betont ebenso in seinem Schlußbericht vom 26. Januar

,Wie bereits im Bericht vom 4. August 1913 genauer ausgeführt wurde, ging eine nicht ganz unbeträchtliche Zahl besonders in den Frühjahrsmonaten und dann plötzlich wieder in der zweiten Hälfte des Monats August interkurrent (zumeist infolge Stallseuchen) ein."

E h r l i c h erklärt dann aber ausdrücklich:

„Selbst bei den mit massivsten Dosen (bis 3,0 ccm der obigen Kulturverdünnung) gespritzten Meerschweinchen ergab der Obduktionsbefund der interkurrent eingegangenen Tiere keine auf die Injektion der Friedmann-Bazillen zurückzuführenden Organveränderungen."

Beiläufig sei erwähnt, daß schon damals u. a. auch L ö f $f$ le r ohne jeden Vorbehalt von der Unschädlichkeit des Mittels überzeugt war. Das geht aus einem Briefe Geh.-Rat $\mathrm{H}$ a ge rs (Magdeburg) (des bekannten Tuberkulinanhängers) hervor, in dem $\mathrm{H}$ a g e $\mathbf{r}$ schreibt:

,Als ich die Absicht hatte, mich über das Friedmannsche Mittel zu orientieren, ging ich vorher zu Herrn Prof. L ö f f l e $\mathrm{r}$ in das Institut für Infektionskrankheiten. Auf meine Frage, ob das Mittel dem Menschen gefährlich sein könne, antwortete er: dasselbe sei dem Menschen ganz ungefährlich; er selbst sei vereit, sich jeden Augenblick dasselbe einverleiben zu lassen."

Ferner schreibt Herr Geh.-Rat Kirchner: „Friedman $n$ machte mir den Vorschlag, seine Fälle anzusehen" und versucht dann seine Ablehnung damit zu begründen, daß er es für unmöglich gehalten habe, „, durch ihre einmalige Besichtigung ein zuverlässiges Urteil über die Behandlung zu gewinnen". Diese Darstellung widerspricht der Wahrheit. Niemals ist an $\mathrm{K}$ i r $\mathrm{ch} \mathrm{n}$ e $\mathbf{r}$ von mir das Ansinnen gestellt worden, $\mathrm{daB}$ er durch eine einmalige Besichtigung ein Urteil gewinnen solle. Vielmehr habe ich $\mathrm{K}$ i r ch n e r wiederholt gebeten, sich Fälle vor, während und nach der $\mathrm{Fr}$ i e d $\mathrm{m}$ a $\mathrm{n} n$ schen $\mathrm{Be}-$ handlung anzusehen. Anfang Februar 1913 hatte ich ihn sogar gebeten, Fälle, die für ihn beweisend seien, selbst auszuwählen oder auswählen zu lassen und mir zur Behandlung zu aberweisen, habe aber die Antwort bekommen, er habe keine Zeit. Das letzte Mal hatte ich ihn Mitte Februar 1914 schriftlich gebeten, am 26. Februar $1914^{1}$ einer größeren Vorführung von Tuberkulösen beizuwohnen, und ihn gleichzeitig gebeten, falls er an diesem Tage verhindert sein sollte, einen anderen ihm genehmen Termin zu nennen.

$\mathrm{K}$ i r c h n e rs Antwort lautete:

Berlin, W. 30. 12. II. 1914. Euer Hochwohlgeboren erwidere Ich auf dle gefällige Zuschrlft vom 9. d. Mts. ergebenst, daß es mir am 26. d. Mts. und an getal fo Zuschrift vom 9. d. Mts. crgebenst, daß es mir am 26. I. Mts. und an
den folgenden Tagen nicht möglich lst, Ihre Kranken zu sehen. In gröBter Hochachtung sehr ergebenst KI r ch ne r."

Er hat es also nicht für notwendig erachtet, sich durch Augenschein von der Wirkung des Mittels zu überzeugen.

Ich bemerke, daß der Amtsnachfolger K i r c hne rs Herr Geh.-Rat Go t t s te in, sehr bald nach seinem Amtsantritt an mich das Ersuchen richtete, eine größere Reihe von Fällen zu sehen.

Was die von $\mathrm{K}$ i r c h n e r zitierte Schrift des Herrn Prof Dührssen anbelangt, so hat $D \ddot{u} h r s s e n$ diese aus eigenster Initiative, und zwar deswegen verfaßt, um, wie er sich ausdrückte, ,doch endlich einmal das der Frie d m a n n schen

1) An diesem Tage waren nämlich die von Kirchner erwähnten Lungenheilanstaltsärzte bei mir in Berlin. Was die Herren zu mir ge. führt hatte, geht am besten aus ihrem an mich gerichteten Vorstands schreiben vom 13. Januar 1914 hervor:

„Wir glauben sagen zu dürfen, daß die Milglieder fast durchweg zu der Ueberzeugung gekommen sind, daß es sich bel Ihrem MIttel um einen ganz neuen wlssenschaftlich gut begründeten und vielversprechenden Weg handelt, durch den vlelleicht die Prophylaxe und therapeutische Behandlung der Tuberkulose und der Skrofulose in entscheidender Weise gefördert und verändert werden kann. Wir sind alle der Meinung, daß $\mathrm{Ihr}$ Mittel in ausgedehntester Weise an einem geeigneten Krankenmaterial und von sachkundiger seite nachgeprüft werden muß. Wir hoffen und erwarten, daB bei diesen Versuchen für die Richtigkeit Ihrer Anschauungen und für die Wirksamkeit Ithres Mittels entscheldende Erfolge Ihrer Anschauungen und făr die Wirksamkeit Ihres Mittels" entscheidende Erfolge
erzielt werden können. . . Da wir nun die Erfahrung gemacht haben, die Sle zweifellos selbst bestätigen werden, daß es vor allen Dingen darauf ankommt, ein neues Tuberkuloseheilmittel wirkllch Im Sinne und nach den Angaben des Erflnders anzuwenden, so haben viele von uns den dringenden Wunsch, von Ihnen oder elnem Ihrer mit lhrer Behandlung und Ihren Ideen völllg vertrauten Mltarbelter In dle Behandlung mit Ihrem Mlttel elngefährt zu werden."
Sache widerfahrene Unrecht darzulegen". Den Vorwurf $\mathrm{K} \mathrm{i} \mathrm{r} \mathrm{c} \mathrm{h} \mathrm{-}$ ne rs, daß er ,von mir und meinen Freunden in wenig vornehmer Weise angegriffen" sei, weise ich zurück. Wenn $\mathrm{K}$ i r c hn e r Herrn Prof. D üh rs sen wegen seiner Behauptung, K i r chner habe die Reinheitsprüfung des Mittels durch L ö f $\mathrm{fle} r$ verboten, der Unwahrheit zeiht, so betone ich demgegenüber nochmals, daß $\mathrm{K}$ i r c h n e $\mathbf{r}$ in der Tat Herrn Geh.Rat $L$ ö f l le r die Reinheitsprüfung des Friedmannschen Mittels durch Versagung seiner hierzu erforderlichen Erlaubnis verboten hat. Herr Geh.-Rat $L$ ö f f le $r$ hatte sich Mitte März 1914 auf Anfrage der Fabrik Dr. Haase \& Co. gegenüber bereit erklärt, die Reinheitskontrolle des Mittels zu übernehmen, vorausgesetzt, daß seine vorgesetzte Behörde es gestatte. Auch Herrn Geh.-Rat $K \mathrm{r}$ a u s gegenüber hatte L ö f fle r diese seine Bereitwilligkeit, die Prüfung zu übernehmen, am 12. März 1914 erklärt. Anfang April erklärte $L$ ö $f$ f l e r jedoch. daß er die Reinheitsprüfung nicht übernehmen könne, da seine vorgesetzte Behörde es nicht gestatte. Die vorgesetzte Behörde L ö f f l e r s war die Medizinalabteilung (Chef: Geh.-Rat Kirchner). Es hat also nicht, wie Kirchner angibt, der im August 1914 ausgebrochene Krieg, sondern sein im März desselben Jahres ergangenes Verbot, die L ö f fle rsche Kontrolle unmöglich gemacht.

Und doch wurde wiederum in der unter $\mathrm{Kirchners}$ Vorsitz veranstalteten Beratung über das Friedmannsche Mittel am 15. Juni 1914 einstimmig beschlossen, daß eine Kontrolle der Herstellung des Mittels, worin selbstverständlich die Reinheitskontrolle eingeschlossen sein mußte, einzuführen sei. Es war also nach außen hin die Herstellungs- und Reinheitskontrolle für notwendig erklärt, jedoch die Ausführung derselben erwiesenermaßen unmöglich gemacht.

Was meine Tätigkeit in Amerika im Frahjahr 1913, die Millionen-Dollarofferte" und die "Mißerfolge" daselbst anbetrifft, so sind alle diese Dinge ja längst einwandfrei widerlegt, so z. B. in dem Artikel des Chefredakteurs des „Berliner LokalAnzeigers", Herrn v. K u p f e r, vom 6. Juli 1913, in welchem auf Grund dokumentarischer Beweise die in derselben Zeitung vom 8. März 1913 über ,mißlungene Schwindsuchtskuren Frie d man $\mathrm{s}$ in Amerika" gemachten Angaben widerrufen sind. $v$. $K$ u p f e $r$ sagt:

Lage und Verleumdung fsind von Amerlka hier herübergetragen und geschrleben und gekabelt worden! Davon habe Ich mlch tiberzeugt, und dartiber aufgeklärt $\mathrm{zu}$ werden hat das zwischen Zwelfel und Hoffnung schwankende Laienpublikum auch el in Recht! "Qewinnsucht" - sprechen wlr es nur aus das war der Vorwurf, der dem Arzt aus dieser Welgerung (das Mittel allgemeln freizugeben), konstrulert, war das Motlv, das nach den Berichten selner Amerlkafahrt unter Entstellung erwlesener Wahrhelten untergeschoben wurde.. Dräben hat man es dann zu bösartlgen Machenschaften - denen slch freilich belspiellose Ehrungen gegenüberstellten -, ausgenü $t z t$, Machenschaften, deren Wirkung selbst hierher reflektlerte. Ein Milllonär sollte Frie d $\mathbf{m}$ a $n \mathbf{n}$ unter fabelhaften Geldangeboten übers Meer gelockt haben. Dieser Mythus blldet die Grundlage des Kampfes gegen Frledmann. Man weiB, wle schwer Mythenbildung zu bekampfes gegen Was lst aber die Wahrheit, wie Ich sie in Dokumenten schwarz kämpfen Ist. Was ist aber die $W$ a h rhe it, wie lch sie in Dokumenten schwarz
auf wel $\beta$ sah!? Auf elgene Kosten reist er. Da kommt das Zusammentreffen auf welB sah!? Auf elgene Kosten reist er. Da kommt das Zusammentreffen mit jenem, ,Millionär", und do $r t$ erst, da n $n$ erst taucht jenes ominose Angebot auf, das Frledmann kllpp und klar, ohne Besinnen zurückwelstl Der ,Millionär" bestätigt nachher diese Zurückweisung schwarz auf welB und lăßt äberdies auch zur Bekrä́ftigung elne Erklärung In glelchem Sinne drucken, dle er selbst zum Ehrenschutz des deutschen Arztes in vlelen Tausenden von Exemplaren verbreltetI $D$ as ist die Wahrheit 1 Das Dokument habe lch gesehen, von

diesem notorischen, einwandfreien Totschlag eines höchstbedent
habe ich aber In den Berlchten von dort hier nichts getesen."

Was die von $\mathrm{K}$ i r c h n e r zitierte Angabe betrifft, „daß von den während $\mathrm{Frie} \mathrm{dmanns}$ Anwesenheit in New York von ihm und seinen Mitarbeitern mit dem Friedmannschen Mittel behandelten Schwindsüchtigen (jetzt werden im ganzen 47 Fälle aufgezählt) somit keine einzige Heilung hätte bestätigt werden können," so stelle ich demgegenüber fest, daß tatsächlich während meiner Anwesenheit in New York von mir und anderen Aerzten mit dem Friedmannschen Mittel nachweislich mehr als 1000 Fälle behandelt worden sind. Uebrigens bin ich keineswegs für alle Behandlungen ,nach Friedmann" und mit "Friedmann-Präparaten" verantwortlich, denn ebenso wie zurzeit hier in Berlin drei Aerzte hinter meinem Rücken unter Mißbrauch meines Namens Präparate vertreiben, so tat dies auch 1913 ein derartiger Arzt in New York. Nach einem mir aus New York vorliegenden Bericht vom 10. Juli 1919 haben auch amerikanische Aerzte, so u. a. Dr. C. M. P o r t e r (Colfax, Jowa), Dr. G. W. D w in ell, Dr. Kellog, Dr. J. J. M a yer (New York), Dr. C. I. M a t h e w s (Spokane, Washington) mit dem Friedmannschen Mittel gute Heilerfolge erzielt. Hiermit ist für mich die Polemik erledigt. Die Dinge sind inzwischen durch die Entwicklung überholt. 